\title{
Quando si tasta con il gusto
}

\author{
Veronica Boschi
}

PUBBLICATO: 16 APRILE 2019

\section{Quesito:}

Alcuni lettori trovano familiare associare il verbo tastare al significato 'assaggiare, gustare', diffuso in molti dialetti italiani, e chiedono delucidazioni su tale uso e sulla sua ammissibilità in italiano.

\section{Quando si tasta con il gusto}

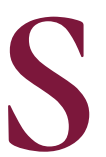

econdo i dizionari dell'italiano, il verbo tastare indica l'azione di 'palpare, toccare esercitando una pressione leggera e ripetuta con la mano, con il piede, o anche con un oggetto, per accertare qualcosa al tatto, per saggiare la qualità e la consistenza di un materiale, ecc.' (GDLI; Devoto-Oli 20r8; GRADIT). Per estensione, il gesto di tastare può alludere anche alla sfera sessuale ed essere inteso nel senso di 'palpare per trarne compiacimento sessuale' (GDLI; Devoto-Oli 20r8; GRADIT; Vocabolario Treccani online).

In genere, oltre all'azione fisica di toccare ripetutamente, il tastare implica l'intenzione di verificare o di provare qualcosa attraverso il tatto, ed è proprio da questa accezione che derivano alcuni usi figurati del termine: per esempio, la locuzione tastare con mano, ovvero 'fare la prova diretta di qualcosa' (GDLI; Devoto-Oli 20I8), o tastare nel significato di 'mettere alla prova qualcuno' (GDLI):

\footnotetext{
Alla fine dell'anno scolastico, il professore compulsi i suoi registri, sommi e divida, dopo aver di nuovo tastato, interrogato, rivisto: cinque e tre quarti, può far l'esame; cinque e un quarto, non può (Giovanni Pascoli, La scuola classica, in Miei pensieri di varia umanitá, Vincenzo Muglia Editore, Messina, 1903, p. 143).
}

Il verbo tastare è usato anche per 'conoscere, sapere, indagare', come nelle espressioni tastare il terreno, 'cercare di conoscere lo stato di una questione' (GDLI; Devoto-Oli 20ı8; DELI; GRADIT; Vocabolario Treccani online), e tastare il polso, che, oltre al significato letterale, può significare anche 'sondare le intenzioni di una persona' (Devoto-Oli 2or8; Garzanti; DELI; GRADIT; Vocabolario Treccani online). Infatti, è comune che i verbi di percezione si prestino a interpretazioni che rielaborano l'esperienza fisico-sensoriale in termini di facoltà conoscitiva, come per esempio vedere per 'capire': "vedo bene che mi sono sbagliato"; "non posso fare come tu vuoi: nonne vedo la ragione" (Vocabolario Treccani online).

Il termine in italiano si riferisce quindi al senso del tatto, si usa principalmente nel significato concreto di 'toccare più volte e leggermente per verificare qualcosa' e nel senso figurato di 'provare qualcosa, testare qualcosa o qualcuno', o anche di 'scoprire, conoscere qualcosa o qualcuno'.

Tra i significati di tastare i dizionari riportano anche un uso antico (GDLI) e regionale (GDLI; GRADIT; Vocabolario Treccani online; Devoto-Oli 20I8) del termine per 'assaggiare, gustare'.

Sulle ragioni per cui la parola ha assunto questo specifico significato, perduto nell'uso italiano, ma molto vivo in alcuni dialetti, sembra voler gettare luce una delle possibili ricostruzioni etimologiche del verbo secondo cui tastare deriverebbe dal latino medievale *tastare, 'conoscere al tatto', esito dell'incrocio di due forme latine tangěre 'toccare' e gustare 'assaggiare' (REW; DEI; DELI; GRADIT; GDLI). Tuttavia, l'etimo resta incerto e discusso, e altre ipotesi non considerano l'interferenza con 
gustare. Una proposta alternativa, meno popolare, ipotizza l'origine dal verbo frequentativo latino volgare "taxitāre 'accarezzare palpando, esplorare', iterativo del latino taxāre 'toccare con forza e ripetutamente' (FEW). L'Etimologico invece presuppone un participio taxitum, in concorrenza con taxatum, direttamente da taxāre, e reputa improbabile la forma iterativa *taxitāre. Flechia nelle Postille etimologiche (I878-I885) considera il verbo *taxitāre un frequentativo di tangěre.

In italiano le sporadiche attestazioni di tastare per riferirsi all'azione di 'assaggiare' si trovano principalmente nella lingua letteraria del 'zoo (GDLI). Se ne attestano alcuni esempi nel Volgarizzamento dellesposizione del Paternostro del fiorentino Zucchero Bencivenni: "Luomo tasta ed assavora il vino innanzi che l'uomo ne bea a sua volontade"; "Che altrimenti conosce il vino ch'il vede in un bello vetro, et altrimenti quelli che ne bea, e tasta, et assavora."; un altro esempio è nella commedia Li vani amori del veneziano Giovanni Francesco Loredano (I588): "Lasciatemi tastare questi capponi".

È controversa invece l'interpretazione del passaggio del Decameron di Boccaccio:

Tastate le botti che si credeva che piene d'olio fossero, trovò quelle esser piene d'acqua marina.

La citazione compare alla voce tastare, 'toccare', nelle prime due edizioni del Vocabolario della Crusca secondo cui il verbo andrebbe inteso nel senso figurato di 'cercare', 'guardare'. Il testo è riportato anche da alcuni recenti dizionari, che interpretano il verbo in modo diverso: il GRADIT intende il termine nel significato di 'saggiare mediante una data operazione', secondo un uso solo letterario; il Vocabolario Treccani online gli attribuisce il significato 'fare prova, fare assaggio di qualche cosa'; il GDLI propone la citazione tra le attestazioni di tastare per 'gustare, assaggiare'.

L'uso di tastare per riferirsi al gusto, oggi desueto in italiano, si è mantenuto vivo fino ai nostri giorni in alcuni dialetti. Per conoscere la diffusione sul territorio italiano delle forme dialettali riconducibili a tastare nel significato di 'gustare' si può consultare la voce 'assaggiare' (carta IO2I) dell'AIS (Atlante linguistico ed etnografico dell'Italia e della Svizzera meridionale). Le aree geografiche interessate sono: l'Italia settentrionale (Liguria occidentale: attastâ, tastấ; Piemonte: tasté; Lombardia meridionale e orientale: tastà; Emilia orientale: tastèr, tastàr, tastà, tastế; Veneto orientale e Trentino: tastàr), la Sicilia (tastari) e la Sardegna (tastare, attastare). Benché il GRADIT consideri questa accezione del termine una forma regionale toscana, secondo l'AIS e i dizionari dell'uso toscano la voce non assume il significato di 'assaggiare' in questa area.

Tra i dialetti dell'Italia meridionale l'uso di tastare per 'gustare' è attestato esclusivamente nel siciliano e nel sardo, per tale ragione si ritiene poco plausibile che si tratti di una parola originaria di queste regioni. Rohlfs (I977) considera il siciliano tastari un tipico settentrionalismo che si sarebbe diffuso successivamente nell'isola. Lorigine galloitalica sembra confermata inoltre dall'assenza della voce dai primi testi siciliani, aperti invece ai normannismi (Varvaro, 2014). In merito al sardo, è probabile che la forma tastare sia stata ereditata dal catalano tastar 'gustare, provare' (Wagner, Dizionario etimologico sardo, Heidelberg, 1964). Infatti, il verbo tastare nel significato di 'assaggiare' è diffuso anche in altre lingue: il catalano e l'aragonese tastar; il francesetâter e il provenzale tastar, attestato dal secolo XII, poi passato all'inglese to taste nel XIV secolo e al basco dastatu (anche nella forma txestatu) nel XVI secolo (FEW; REW; Arbelaiz, I978; Varvaro 2014; Gioeni, I885; Elhuyar Hiztegia, 2006).

Oggi in Francia, come in Italia, tâter 'assaggiare' è meno frequente di tâter 'toccare': corrisponde a un uso antico ed è vitale prevalentemente nei dialetti del mezzogiorno (Dictionnaire de l'Académie française, $8^{\mathrm{e}}$ édition,I935; Varvaro, 2014). Litaliano e il francese nel tempo hanno privilegiato il 
significato relativo al senso del tatto, l'inglese invece ha seguito un percorso di selezione semantica simile, ma inverso: taste, che oggi si riferisce esclusivamente all'azione di'assaggiare', aveva in origine tra i suoi significati quello di 'esaminare al tatto', 'sentire attraverso il tatto', oggi obsoleto (Jess Stein, The random house dictionary of the english language, Laurence Urdang Editor, New York, I97I; J. Murray, H. Bradely, W.A. Craigie, C.T. Onions, A new english dictionary of historical principles, Occoford, Clarendon Press, I9I9). Anche lo spagnolo registra la voce tastar con il doppio significato di 'toccare' e 'gustare', seppur classificandola come desueta (Diccionario de la lengua española, 23. edición, 20I4).

In conclusione, sebbene tastare in italiano sia da usare in riferimento al tatto, è indubbio che in origine il verbo fosse impiegato per riferirsi a entrambi i sensi (tatto e gusto), come dimostra la storia del termine e come si registra in alcuni dialetti d'Italia (Liguria, Lombardia, Emilia-Romagna, Veneto e Trentino) in cui tastare è ancora oggi usato per riferirsi sia al tatto che al gusto. Nel tempo l'uso dialettale del verbo è filtrato nell'italiano regionale ed è probabile che tale passaggio abbia alterato la percezione di alcuni parlanti, creando sovrapposizioni con la forma italiana. In fondo, ciò che il verbo tastare esprime, che si tratti di 'toccare leggermente e ripetutamente' o di 'assaggiare', è l'idea di 'provare qualcosa', di 'saggiare' attraverso il tatto o il gusto, ovvero usando due canali per mezzo dei quali è possibile sottoporre un oggetto, un materiale o anche una persona, in modo figurato, a prove al fine di verificarne e sperimentarne le qualità, le caratteristiche, le attitudini, le doti.

Nota bibliografica:

- Juan José Arbelaiz, Las etimologias vascas en la obra de Luis Michelena, Kardaberaz Bilduma 3I, Tolosa, 1978.

- Elhuyar Hiztegia, Euskara-Gaztelania. Castellano-Vasco, Usurbil, Elhuyar, 2006.

- Giovanni Flechia, Postille etimologiche. Recensione di Saggio di un glossario modenese ossia studii del conte Giovanni Galvani intorno le probabili origini di alquanti idiotismi della cittá di Modena e del suo contado "Archivio Glottologico Italiano", I878-1885.

- Giuseppe Gioeni, Etimologie siciliane, Tipografia dello statuto, Palermo, I885.

- Gerhard Rohlfs, Supplemento ai vocabolari siciliani, München, I977.

- Alberto Varvaro, Vocabolario storico-etimologico del siciliano, Centro Studi Filologici, Palermo, 2014.

- Max Leopold Wagner, Dizionario Etimologico Sardo, Carl Winter, Heidelberg, 1964

\author{
Cita come: \\ Veronica Boschi, Quando si tasta con il gusto, "Italiano digitale", 2019, IX, 2019/2 (aprile-giugno) \\ DOI: $10.35948 / 2532-9006 / 2020.3108$ \\ Copyright 2019 Accademia della Crusca \\ Pubblicato con licenza creative commons CC BY-NC-ND
}

